BULL. AUSTRAL. MATH. SOC.

VOL. 14 (1976), 409-416.

\title{
Certain classes of univalent functions with negative coefficients
}

\section{V.P. Gupta and P.K. Jain}

The subclasses $S^{*}(\alpha, \beta)$ and $C^{*}(\alpha, \beta)$ of $T$, the class of analytic and univalent functions of the form $f(z)=z-\sum_{n=2}^{\infty}\left|a_{n}\right| z^{n}$ have been considered. Sharp results concerning coefficients, distortion of functions belonging to $S^{*}(\alpha, \beta)$ and $C^{*}(\alpha, \beta)$ are determined along with a representation formula for the functions in $S^{*}(\alpha, \beta)$. Furthermore, it is shown that the classes $S^{*}(\alpha, \beta)$ and $C^{*}(\alpha, \beta)$ are closed under arithmetic mean and convex linear combinations.

\section{Introduction}

Let $S$ denote the class of functions of the form equal to $z+\sum_{n=2}^{\infty}\left|a_{n}\right| z^{n}$ that are analytic and univalent in the unit disk $|z|<1$. We denote by $S(\alpha)$ and $C(\alpha)$ the subclasses of $S$ consisting of functions which are, respectively, starlike of order $\alpha$ and convex of order $\alpha$. A function $f \in S$ is in $S(\alpha, \beta)$, the class of starlike functions of order $\alpha(0 \leq \alpha<1)$ and type $\beta(0<\beta \leq 1)$ if and only if

$$
\left|\left[\frac{z f^{\prime}(z)}{f(z)}-1\right] /\left[\frac{z f^{\prime}(z)}{f(z)}+(1-2 \alpha)\right]\right|<\beta,(|z|<1) .
$$

Received 23 February 1976. 
Further, $f \in S$ is in $C(\alpha, \beta)$, the class of convex functions of order $\alpha$ and type $\beta$ if and only if $z f^{\prime}(z) \in S(\alpha, \beta)$.

Let $T$ denote the subclass of $S$ consisting of functions whose nonzero coefficients, from the second on, are negative; that is, an analytic and univalent function $f$ is in $T$ if and only if it can be expressed as $f(z)=z-\sum_{n=2}^{\infty}\left|a_{n}\right| z^{n}$. We denote by $S^{*}(\alpha), C^{*}(\alpha), S^{*}(\alpha, \beta), C^{*}(\alpha, \beta)$ the classes obtained by taking intersections, respectively, of the classes $S(\alpha), C(\alpha), S(\alpha, \beta)$, and $C(\alpha, \beta)$ with $T$. In [1], Schild considered a subclass of $T$ consisting of polynomials having $|z|=1$ as radius of univalence. For this class, he obtained a necessary and sufficient condition in terms of the coefficients, and with the aid of this he derived better results for certain quantities connected with the conformal mapping of univalent functions. Silverman [2] determined coefficient inequalities, distortion, and covering theorems for the classes $S^{*}(\alpha)$ and $C^{*}(\alpha)$.

In this paper, sharp results concerning coefficients and distortion theorems for the classes $S^{*}(\alpha, \beta)$ and $C^{*}(\alpha, \beta)$ are determined. We also show that these classes are closed under 'arithmetic mean' and 'convex linear combinations'.

\section{Coefficients theorems}

THEOREM 1. A function $f(z)=z-\sum_{n=2}^{\infty}\left|a_{n}\right| z^{n}$ is in $S^{*}(\alpha, \beta)$ if and only if

$$
\sum_{n=2}^{\infty}\{(n-1)+\beta(n+1-2 \alpha)\}\left|a_{n}\right| \leq 2 \beta(1-\alpha) .
$$

This result is sharp.

Proof. Let $|z|=1$. Then 


$$
\begin{aligned}
\left|z f^{\prime}(z)-f(z)\right|-\beta \mid z f^{\prime}(z) & +(1-2 \alpha) f(z) \mid \\
& =\left|\sum_{n=2}^{\infty}(1-n)\right| a_{n}\left|z^{n}\right|-\beta\left|2(1-\alpha) z-\sum_{n=2}^{\infty}(n+1-2 \alpha)\right| a_{n}\left|z^{n}\right| \\
& \leq \sum_{n=2}^{\infty}\{(n-1)+\beta(n+1-2 \alpha)\}\left|a_{n}\right|-2 \beta(1-\alpha) \leq 0 .
\end{aligned}
$$

Hence, by the maximum modulus theorem, $f \in S^{*}(\alpha, \beta)$.

For the converse, assume that

$$
\begin{aligned}
\left|\left[\frac{z f^{\prime}(z)}{f(z)}-1\right] /\left[\frac{z f^{\prime}(z)}{f(z)}+(1-2 \alpha)\right]\right| & =\frac{\sum_{n=2}^{\infty}(n-1)\left|a_{n}\right| z^{n}}{2(1-\alpha) z-\sum_{n=2}^{\infty}(n+1-2 \alpha)\left|a_{n}\right| z^{n}} \\
& <\beta,(|z|<1) .
\end{aligned}
$$

Since $|\operatorname{Re}(z)| \leq|z|$ for all $z$, we have

$$
\operatorname{Re}\left\{\frac{\sum_{n=2}^{\infty}(n-1)\left|a_{n}\right| z^{n}}{2(1-\alpha) z-\sum_{n=2}^{\infty}(n+1-2 \alpha)\left|a_{n}\right| z^{n}}\right\}<B .
$$

Choose values of $z$ on the real axis so that $\left(z f^{\prime}(z) / f(z)\right)$ is real. Upon clearing the denominator in (2.1) and letting $z \rightarrow 1$ through real values, we obtain

$$
\sum_{n=2}^{\infty}(n-1)\left|a_{n}\right| \leq \beta\left\{2(1-\alpha)-\sum_{n=2}^{\infty}(n+1-2 \alpha)\left|a_{n}\right|\right\} .
$$

This gives the required condition.

Finally, the function

$$
f(z)=z-\sum_{n=2}^{\infty} \frac{2 \beta(1-\alpha)}{(n-1)+\beta(n+1-2 \alpha)} z^{n},
$$

is an extremal function for the theorem.

COROLLARY. $T=S^{*}(0,1)$.

THEOREM 2. A function $f(z)=z-\sum_{n=2}^{\infty}\left|a_{n}\right| z^{n}$ is in $c^{*}(\alpha, \beta)$ if and only if 


$$
\sum_{n=2}^{\infty} n\{(n-1)+\beta(n+1-2 \alpha)\}\left|a_{n}\right| \leq 2 \beta(1-\alpha) .
$$

Proof. It immediately follows by appealing to Theorem 1 .

\section{A representation formula}

We now proceed to prove a theorem which gives a representation for functions in $S^{*}(\alpha, \beta)$.

THEOREM 3. A function $f(z)=z-\sum_{n=2}^{\infty}\left|a_{n}\right| z^{n}$ is in $S^{*}(\alpha, \beta)$ if and only if

$$
f(z)=z \exp \left\{2(1-\alpha) \int_{0}^{z} \varphi(t) /\left(1-t_{\varphi}(t)\right) d t\right\},
$$

where $\varphi(z)$ is analytic and satisfies $|\varphi(z)| \leq \beta$, for $|z|<1$.

Proof. Let $f \in S^{*}(\alpha, \beta)$. Then

$$
\left|\left[\frac{z f^{\prime}(z)}{f(z)}-1\right] /\left[\frac{z f^{\prime}(z)}{f(z)}+(1-2 \alpha)\right]\right|<\beta,(|z|<1) .
$$

Since the absolute value vanishes for $z=0$, we have

$$
\left(\frac{z f^{\prime}(z)}{f(z)}-1 / \frac{z f^{\prime}(z)}{f(z)}+(1-2 \alpha)\right)=V(z),
$$

where $V(z)$ is analytic and $|V(z)| \leq \beta$, for $|z|<1$. The 'only if' part is easily obtained by integrating (3.2) with $V(z)=z \varphi(z)$, and the other part by differentiating (3.1).

\section{Distortion theorems}

THEOREM 4. If $f \in S^{*}(\alpha, \beta)$, then

(4.1) $\quad r-\frac{2 \beta(1-\alpha)}{1+\beta(3-2 \alpha)} r^{2} \leq|f(z)| \leq r+\frac{2 \beta(1-\alpha)}{1+\beta(3-2 \alpha)} r^{2}, \quad(|z|=r)$

and

(4.2) $\quad 1-\frac{4 \beta(1-\alpha)}{1+\beta(3-2 \alpha)} r \leq\left|f^{\prime}(z)\right| \leq 1+\frac{4 \beta(1-\alpha)}{1+\beta(3-2 \alpha)} r, \quad(|z|=r)$.

Proof. In view of Theorem 1, we have 


$$
\begin{aligned}
1+\beta(3-2 \alpha) \sum_{n=2}^{\infty}\left|a_{n}\right| & \leq \sum_{n=2}^{\infty}\{(n-1)+\beta(n+1-2 \alpha)\}\left|a_{n}\right| \\
& \leq 2 \beta(1-\alpha)
\end{aligned}
$$

which gives

$$
\sum_{n=2}^{\infty}\left|a_{n}\right| \leq \frac{2 \beta(1-\alpha)}{1+\beta(3-2 \alpha)}
$$

Therefore

$$
|f(z)| \leq r+\sum_{n=2}^{\infty}\left|a_{n}\right| r^{n} \leq r+\frac{2 \beta(1-\alpha)}{1+\beta(3-2 \alpha)} r^{2}
$$

and

$$
|f(z)| \geq r-\sum_{n=2}^{\infty}\left|a_{n}\right| r^{n} \geq r-\frac{2 \beta(1-\alpha)}{1+\beta(3-2 \alpha)} r^{2} .
$$

Hence (4.1) follows.

Further

$$
\left|f^{\prime}(z)\right| \leq 1+\sum_{n=2}^{\infty} n\left|a_{n}\right||z|^{n-1} \leq 1+r \sum_{n=2}^{\infty} n\left|a_{n}\right|
$$

and

$$
\left|f^{\prime}(z)\right| \geq 1-\sum_{n=2}^{\infty} n\left|a_{n}\right||z|^{n-1} \geq 1-r \sum_{n=2}^{\infty} n\left|a_{n}\right| .
$$

Also, from Theorem 1, we note that

$$
\sum_{n=2}^{\infty} n\left|a_{n}\right| \leq \frac{4 \beta(1-\alpha)}{1+\beta(3-2 \alpha)} \text {. }
$$

Hence (4.2) follows also.

REMARK. The bounds in (4.1) and (4.2) are sharp, since the equalities are attained for the functions:

$$
f(z)=z-\frac{2 \beta(1-\alpha)}{1+\beta(3-2 \alpha)} z^{2}, \quad(z= \pm r) .
$$

Using the same technique as in the proof of Theorem 4 we obtain the following: 
THEOREM 5. If $f \in C^{*}(\alpha, \beta)$, then

$$
r-\frac{\beta(1-\alpha)}{1+\beta(3-2 \alpha)} r^{2} \leq|f(z)| \leq r+\frac{\beta(1-\alpha)}{1+\beta(3-2 \alpha)} r^{2}, \quad(|z|=r)
$$

and

$$
1-\frac{2 \beta(1-\alpha)}{1+\beta(3-2 \alpha)} r \leq\left|f^{\prime}(z)\right| \leq 1+\frac{2 \beta(1-\alpha)}{1+\beta(3-2 \alpha)} r, \quad(|z|=r)
$$

with equalities for $f(z)=z-\beta(1-\alpha)[1+\beta(3-2 \alpha)]^{-1} z^{2}, \quad(z= \pm r)$.

THEOREM 6. Let $f \in S^{*}(\alpha, \beta)$. Then the disk $|z|<1$ is mapped onto a domain that contains the disk $|\omega|<(1+\beta) / 1+\beta(3-2 \alpha)$. The result is sharp with extremal function $z-2 \beta(1-\alpha)[1+\beta(3-2 \alpha)]^{-1} z^{2}$.

THEOREM 7. Let $f \in C^{*}(\alpha, \beta)$. Then the disk $|z|<1$ is mapped onto $a$ domain that contains the disk $|w|<(1+\beta(2-\alpha)) / 1+\beta(3-2 \alpha)$. The result is sharp with extremal function $z-\beta(1-\alpha)[1+\beta(3-2 \alpha)]^{-1} z^{2}$.

Proofs of Theorems 6 and 7. Proofs follow by letting $r \rightarrow 1$ in (4.1) and $(4.3)$.

\section{Closure theorems}

In this section, we shall prove that the classes $S^{*}(\alpha, \beta)$ and $C^{*}(\alpha, \beta)$ are closed under 'arithmetic mean' and 'convex linear combinations'.

THEOREM 8. If $f(z)=z-\sum_{n=2}^{\infty}\left|a_{n}\right| z^{n}$ and $g(z)=z-\sum_{n=2}^{\infty}\left|b_{n}\right| z^{n}$ are in $S^{*}(\alpha, \beta)$, then $h(z)=z-\frac{1}{2} \sum_{n=2}^{\infty}\left|a_{n}+b_{n}\right| z^{n}$ is also in $S^{*}(\alpha, \beta)$.

Proof. The proof follows directly by appealing to Theorem 1 . In fact, $f$ and $g$ being in $S^{*}(\alpha, \beta)$, we have

$$
\sum_{n=0}^{\infty}\{(n-1)+\beta(n+1-2 \alpha)\}\left|a_{n}\right| \leq 2 \beta(1-\alpha)
$$

and

$$
\sum_{n=2}^{\infty}\{(n-1)+\beta(n+1-2 \alpha)\}\left|b_{n}\right| \leq 2 \beta(1-\alpha) .
$$


It is sufficient, for $h$ to be a member of $S^{*}(\alpha, \beta)$, to show

$$
\frac{1}{2} \sum_{n=2}^{\infty}\{(n-1)+\beta(n+1-2 \alpha)\}\left|a_{n}+b_{n}\right| \leq 2 \beta(1-\alpha),
$$

which will follow immediately by the use of (5.1) and (5.2).

THEOREM 9. Let

$$
f_{1}(z)=z, f_{n}(z)=\frac{2 \beta(1-\alpha)}{(n-1)+\beta(n+1-2 \alpha)} z^{n}, \quad(n=2,3, \ldots) .
$$

Then $f \in S^{*}(\alpha, \beta)$ if and only if it con be expressed in the form $f(z)=\sum_{n=1}^{\infty} \lambda_{n} f_{n}(z)$, where $\lambda_{n} \geq 0$ and $\sum_{n=1}^{\infty} \lambda_{n}=1$.

Proof. Suppose

$$
\begin{aligned}
f(z) & =\sum_{n=1}^{\infty} \lambda_{n} f_{n}(z) \\
& =z-\sum_{n=2}^{\infty} \frac{2 \beta(1-\alpha)}{(n-1)+\beta(n+1-2 \alpha)} \lambda_{n} z^{n} .
\end{aligned}
$$

Then

$$
\sum_{n=2}^{\infty}\left\{\frac{(n-1)+\beta(n+1-2 \alpha)}{2 \beta(1-\alpha)} \lambda_{n} \frac{2 \beta(1-\alpha)}{(n-1)+\beta(n+1-2 \alpha)}\right\} \leq 1 .
$$

Thus, by Theorem 1, $f \in S^{*}(\alpha, \beta)$.

Conversely, suppose $f \in S^{*}(\alpha, \beta)$. Again, by Theorem 1, we have

$$
\left|a_{n}\right| \leq \frac{2 \beta(1-\alpha)}{(n-1)+\beta(n+1-2 \alpha)}, \quad(n=2,3, \ldots) .
$$

Setting

$$
\lambda_{n}=\frac{(n-1)+\beta(n+1-2 \alpha)}{2 \beta(1-\alpha)}\left|a_{n}\right|, \quad(n=2,3, \ldots)
$$

and

$$
\lambda_{1}=1-\sum_{n=2}^{\infty} \lambda_{n}
$$

we have 


$$
f(z)=\sum_{n=1}^{\infty} \lambda_{n} f_{n}(z)
$$

This completes the proof.

The analogues of Theorems 8 and 9 for the class $C^{*}(\alpha, \beta)$ are:

THEOREM 10. If $f(z)=z-\sum_{n=2}^{\infty}\left|a_{n}\right| z^{n}$ and $g(z)=z-\sum_{n=2}^{\infty}\left|b_{n}\right| z^{n}$

are in $C^{*}(\alpha, \beta)$, then $h(z)=z-\frac{1}{2} \sum_{n=2}^{\infty}\left|a_{n}+b_{n}\right| z^{n}$ is also in $C^{*}(\alpha, \beta)$.

THEOREM 11. Let

$$
f_{1}(z)=z, f_{n}(z)=z-\frac{2 \beta(1-\alpha)}{n\{(n-1)+\beta(n+1-2 \alpha)\}} z^{n}, \quad(n=2,3, \ldots) .
$$

Then $f \in C^{*}(\alpha, \beta)$ if and only if it can be expressed in the form $f(z)=\sum_{n=1}^{\infty} \lambda_{n} f_{n}(z)$ where $\lambda_{n} \geq 0$ and $\sum_{n=1}^{\infty} \lambda_{n}=1$.

\section{References}

[1] A. Schild, "On a class of functions schlicht in the unit circle", Proc. Amer. Math. Soc. 5 (1954), 115-120.

[2] Herb Silverman, "Univalent functions with negative coefficients", Proc. Amer. Math. Soc. 51 (1975), 109-116.

\footnotetext{
M.M.H. College,

University of Meerut,

Ghaziabad,

Uttar Pradesh,

India;

Department of Mathematics,

Hans Raj College,

Delhi,

India.
} 